\title{
LAS DIFERENCIAS ENTRE LA EDUCACIÓN A DISTANCIA Y VIRTUAL EN COLOMBIA
}

\author{
The differences between distance and virtual education in Colombia
}

SANDRA NARANJO [1]

Recibido:12 de abril de 2021. Aceptado:12 de junio de 2021

DOI: http://dx.doi.org/10.21017/rimci.2021.v8.n16.a106

\begin{abstract}
Resumen
El articulo presenta las diferencias conceptuales en torno a las modalidades educativas a distancia y virtual en Colombia, analizando el contexto legal bajo la Ley 30 de 1992 y el Decreto 1295 de 2010 que estableció dos modalidades en la educación a distancia: la distancia tradicional y la virtual, además el Decreto 1075 de 2015 y 1280 de 2018 como marco reglamentario de la educación superior y sus modalidades, por otra parte, se hace un recorrido histórico resaltando la incidencia del desarrollo tecnológico, el uso de las TIC, oferta de programas e indicadores de evaluación de la calidad de ambas modalidades.
\end{abstract}

Palabras clave. Educación a distancia; Educación virtual; Plataformas educativas, TIC.

\begin{abstract}
The article presents the conceptual differences around distance and virtual educational modalities in Colombia, analyzing the legal context under Law 30 of 1992 and Decree 1295 of 2010 that established two modalities in distance education: traditional distance and distance education. In addition, Decree 1075 of 2015 and 1280 of 2018 as a regulatory framework for higher education and its modalities, on the other hand, a historical journey is made highlighting the incidence of technological development, the use of ICT, offer of programs and indicators evaluation of the quality of both modalities.
\end{abstract}

Keywords. Distance education; Virtual education; Educational platforms, ICT.

\section{INTRODUCCIÓN}

U na de las principales discusiones que se dan en torno a las modalidades educativas a distancia y virtual, en Colombia, es precisamente la diferencia que existe entre estas y las características que las definen.

La Ley 30 de 1992, por el cual se organiza el servicio público de la Educación Superior, les otorga a la educación presencial y a la educación a distancia el carácter de metodologías; específicamente a la segunda, en el Artículo 15: "las instituciones de Educación Superior podrán adelantar programas en la metodología de educación abierta y a distancia, de conformidad con la presente Ley"; de lo que se deduce que existen dos metodologías de formación educativa: presencial y a distancia; posteriormente, los decretos 2566 de 2003 y 2755 de 2006 sentaron bases regulatorias y definición de características de la educación a distancia, y el Decreto 1295 de 2010 estableció dos modalidades en la educación a distancia: la distancia tradicional y la virtual [1].

1 Docente-investigadora de la Corporación Universitaria Republicana y miembro del grupo de investigación Grupo de Investigación y desarrollo para la Innovación Sostenible (GIDIS). ORCID: https://orcid.org/0000-0001-9979-8654. Correo electrónico: snaranjo@urepublicana.edu.co

2 Por el cual se proponía reglamentar el Sistema de Aseguramiento de la Calidad de la Educación Superior, el registro calificado de que trata la Ley 1188 de 2008 y los artículos 53 y 54 de la Ley 30 de 1992 sobre acreditación, por lo que se subrogan los Capítulos 2 y 7 del Título 3 de la Parte 5 del Libro 2 del Decreto 1075 de 2015 -Único Reglamentario del Sector Educación, y se deroga por tanto el Decreto 1295 de 2010; pero, que no fue aprobado porque ni superó la fase de discusiones. 
Posteriormente, el Decreto 1075 de 2015 [2], Único Reglamentario de la Educación Superior del Sector Educación, estableció la definición de programas a distancia, como "aquellos cuya metodología educativa se caracteriza por utilizar estrategias de enseñanza-aprendizaje que permiten superar las limitaciones de espacio y tiempo entre los actores del proceso educativo" (Artículo 2.5.3.2.6.1) y los programas virtuales como aquellos que "adicionalmente, exigen el uso de las redes telemáticas como entorno principal, en el cual se lleven a cabo todas o al menos el ochenta por ciento $(80 \%)$ de las actividades académicas" (Artículo 2.5.3.2.6.2.), y la propuesta de Decreto 1280 del 25 de julio de 2018[2], establece con respecto a los programas a distancia que:

La educación a distancia es una modalidad educativa que trasciende los espacios físicos para el desarrollo de los procesos de enseñanza y aprendizaje enmarcados en un contexto institucional. Se caracteriza por la separación en el tiempo y el espacio entre el docente y el estudiante; el uso de medios y recursos tecnológicos; comunicación bidireccional, que puede incluir tutorías, y que propende porque el estudiante sea el principal impulsor de su aprendizaje" (Artículo 2.5.3.2.2.9.1., Sección 2: Registro calificado. Subsección 9. Programas a distancia y virtuales) [3].

\section{En tanto los programas virtuales:}

Se caracterizan por la superación espacio-temporal y exigen, además de lo anterior, el uso de las redes telemáticas, las TIC y el ciberespacio como entorno principal en el cual se lleven a cabo todas o, al menos, el 80\% de las actividades académicas. / Los programas virtuales pueden adoptar prácticas de enseñanza y aprendizaje tradicionales en los que, típicamente, se da una combinación de las interacciones cara a cara mediante el aprendizaje en línea. / En todos los casos, es necesario que la proporción de cursos o módulos combinados de estos programas sea claramente explicada y divulgada en la oferta que se haga al público y previamente a cualquier proceso de inscripción o matrícula. / De igual manera, es necesario que el diseño curricular explicite la forma cómo se da la necesaria articulación entre los componentes presenciales y los virtuales, incluyendo también lo relativo a las actividades de evaluación (Artículo 2.5.3.2.2.9.2, Sección 2: Registro calificado. Subsección 9. Programas a distancia y virtuales) [3].

\section{UN ASUNTO DE HISTORIA}

La discusión sobre sus diferencias encuentra un punto de resolución cuando se consideran los procesos históricos de la modalidad a distancia tradicional y de la modalidad virtual en el ámbito mundial y en el ámbito nacional

De acuerdo con el Ministerio de Educación Nacional, en un documento de discusión sobre los lineamientos y condiciones de calidad para programas académicos en modalidades virtual y combinada, la educación a distancia tiene sus orígenes en la educación por correspondencia que surgió en Estados Unidos, a mediados de 1850, y que se formalizó en una oferta de programas universitarios de pregrado y posgrado, en la Illinois Wesleyan University, en 1874; de forma paralela surgieron en el resto del mundo programas y ofertas de educación a distancia; en Canadá, en 1889; en Oceanía, en 1910; en Europa y América Latina, en 1939, y en África y Asia, en 1946 [4]. La evolución de los medios de comunicación de la segunda revolución industrial, los medios masivos de comunicación, y posteriormente, los medios de comunicación de la llamada tercera revolución industrial, el computador y la internet, determinaron el desarrollo de entre tres y cuatro etapas o generaciones en la educación a distancia [4] [5].

La educación por correspondencia evolucionó al ritmo de las innovaciones, y así su formato se fue adaptando a las nuevas oportunidades que representaban las tecnologías emergentes. Las primeras modalidades de educación a distancia se basaban fundamentalmente en materiales impresos y enviados por correo postal, sin utilizar prácticamente ninguna TIC. La primera generación en adoptar herramientas tecnológicas para la educación a distancia incorporó el teléfono y la televisión. La segunda generación incorporó otros medios, como la transmisión por fax, las cintas de audio y los vídeos. La tercera generación utilizó ante todo los ordenadores, ampliando las posibilidades de impartir enseñanza. Por último, la aparición de Internet y de las tecnologías de banda ancha supuso la cuarta generación de la educación a distancia, que trajo consigo nuevas posibilidades[6].

En términos temporales, y de forma aproximada, la primera generación va hasta la década de 
los 60 y se caracteriza por el uso de material didáctico impreso y la conformación de la modalidad educativa a distancia; la segunda generación, de enseñanza multimedia, emplea fundamentalmente la radio, la televisión y otros medios de reproducción visual como las diapositivas y las retroproyecciones y se ubica en la década de los 70; la tercera generación, de enseñanza telemática, se centra en el uso del computador y ocupa la década de los 80, y la cuarta generación, que coincide con el surgimiento de la educación virtual, depende del uso de redes de computadores como internet, de las redes de la comunicación digital y del desarrollo de plataformas de gestión del aprendizaje (LMS, por sus siglas en inglés de Learning Management System) [4][7].

La época de mayor desarrollo de la educación a distancia en las universidades en el mundo fue entre finales de los setenta y los ochenta; en la mayoría de los países con el apoyo estatal y con la finalidad de contribuir a una mayor cobertura en educación superior y a brindar condiciones de equidad y de inclusión social [1], en esta época se destacan los aportes a los modelos educativos de la educación a distancia de la Open University Británica, del Reino Unido, que sustentó las bases de la enseñanza telemática (recursos educativos impresos, de audio, de video y emisiones de radio y televisión), y en el mundo de habla hispana los aportes de la Universidad Nacional de Educación a Distancia (UNED), y es precisamente este modelo telemático el que se identifica con la educación a distancia tradicional, incluso con el uso de recursos de la comunicación y educación digital propios de la cuarta generación.

En Colombia, la trayectoria de la educación a distancia es de larga data, pues inicia con las escuelas radiofónicas de la Acción Cultura Popular (ACPO), en 1947, orientadas a la población campesina y su cultura, y que se mantuvo vigente por casi 50 años con la integración de "toda una asociación de medios para el aprendizaje: Cadena de Radio Sutatenza, Periódico El Campesino, Biblioteca básica del campesino, discoestudio, casetes de audio, cartillas, video y el Institu to de Formación de Líderes Campesinos del Valle de Tenza" [8].

Recién inaugurada la televisión, el Fondo de Capacitación Popular (1954), ofreció programas de primaria y bachillerato con apoyo televisivo. En
1958 el Ministerio de Educación Nacional abrió programas de capacitación al magisterio, utilizando estrategias de educación a distancia. Para 1968 la capacitación docente se hizo con base en el apoyo televisivo del Canal 11 (hoy Señal Colombia). A través de la Radiodifusora Nacional (1968) se ofreció también el bachillerato por radio. La División educativa de INRAVISIÓN (1975) crea el programa de coproducción de material didáctico para programas de educación básica y de bachillerato. El SENA se une a esta tendencia y en 1977 pone en funcionamiento el Centro de Formación a Distancia [1].

La formación universitaria a distancia tuvo un primer movimiento en los primeros años de la década de los 70, orientado a licenciaturas y apoyado por el Instituto Colombiano de Fomento de la Educación Superior (Icfes); en 1982, Colombia adoptó la educación a distancia como política educativa nacional, se creó el Consejo Nacional de Educación Abierta y a Distancia, se le delegaron al Icfes funciones de promoción y evaluación de programas a distancia, y se convirtió la Unidad Universitaria del Sur (Unisur) en la Universidad Nacional Abierta y a Distancia (UNAD) [1];

Pero, fue precisamente la adopción de la política nacional de educación a distancia en 1982, materializada en el establecimiento del Subsistema de Educación a Distancia lo que creo la fractura entre las experiencias del país en la educación a distancia telemática, un desarrollo continuo de la educación a distancia y el surgimiento de la educación virtual; según Facundo

Haber sido ofrecida la modalidad a distancia como un programa prioritario de gobierno podrían llevar a pensar en un auspicioso desarrollo. Sin embargo, la realidad ha sido otra. El subsistema de educación a distancia surge oficialmente en el año 1982, como una "alternativa" ante la imposibilidad del sistema de educación existente de lograr el cubrimiento de la demanda existente. Las nuevas instituciones y programas de la modalidad a distancia que se crearon, debieron someterse al ordenamiento jurídico del Sistema de Educación Postsecundaria establecido por el Decreto-Ley 80 de 1980 y a la política de focalizarse prioritariamente en programas de nivel técnico y tecnológico. En el surgimiento del Subsistema de Educación a Distancia, un hecho es relevante: 
simultáneamente con su creación, desaparecieron los anteriores programas de educación a distancia no formal, tanto la radio y TV educativas oficiales e igualmente el programa ACPO [9].

Con este, se generó un "nuevo modelo" de educación a distancia que supuso no solo la desaparición de los programas a distancia formales y no formales, sino también el retroceso del modelo de enseñanza telemático con el consecuente desaprovechamiento de los recursos educativos de la radio y la televisión, así como de los espacios que se habían conquistado para esta en el Ministerio de Comunicaciones y cuya destinación terminó siendo comercial.

En esta década, pocas universidades continuaron apostándole a esta modalidad educativa, lo que fue el escenario en el que se empezaron a implementar la tercera y cuarta generación de la modalidad a distancia, así como la modalidad virtual.

La educación virtual y a distancia en Colombia surgió en la década de los 90 con el auge de las nuevas tecnologías de la comunicación que imperan en el mundo. Estas tecnologías fueron interpretadas en la educación bajo un plan de masificación de la educación; por lo tanto, la prioridad, originalmente, fue la cobertura de las TIC, garantizando una mayor distribución de las nuevas tecnologías en los colegios, universidades y regiones apartadas del país. Actualmente, el Ministerio de Educación Nacional está trabajando en proyectos de apoyo con base en las TIC, estableciendo lineamientos de calidad y políticas para el fortalecimiento de los programas virtuales especialmente en la educación superior.

Colombia ha atravesado por una etapa de desarrollo e investigación tecnológico-educativa, a partir del cual las instituciones se han preocupado por adquirir tecnología de punta que soporte sus programas y ambientes virtuales de aprendizaje, pero su mayor trabajo ha estado enfocado a establecer modelos pedagógicos para estos, sustentados en las teorías del aprendizaje y de la educación, y en el establecimiento de lineamientos y estándares de calidad para el diseño de contenidos y materiales en español, y sistemas de evaluación, con el objetivo de fortalecer los programas ofrecidos actualmente y desarrollar más programas de educación continuada, pero especialmente de pregrado y postgrado a distancia y virtuales [8].

La educación virtual colombiana se ha convertido en una alternativa de estudio y capacitación para poblaciones que geográficamente se encuentran dispersas. Igualmente, es una opción que está tomando fuerza en las ciudades, ya que se está tomando en cuenta como alternativa a la educación presencial [10].

A mitad de los noventa la modalidad a distancia recupera fuerzas e importancia en los ámbitos nacional e internacional, y surge la Asociación Colombiana de Educación a Distancia, con la finalidad de integrar a las diversas instituciones de educación superior nacionales con programas de educación a distancia y virtuales en torno a estrategias de promoción y mejoramiento de la calidad.

Entre el 1997 y el 98 se inician los primeros programas nacionales de educación virtual (...). Para 2008, Colombia contaba con 44 instituciones que ofrecían programas de e-learning, reuniendo un total de 170 programas de diversa índole, nivel y metodología, así como un alumnado total de 144 605 estudiantes. Ello significaba el 10.05\% de las matriculas en educación superior. Entre las instituciones colombianas con programas de e-learning más resaltantes se cuenta con los casos de la Universidad Nacional Abierta y a Distancia, que hasta la actualidad concentra la mitad de las matrículas en esta modalidad [6].

\section{OfERTA DE EDUCACIÓN A DISTANCIA Y VIRTUAL EN COLOMBIA}

Para 2014, y de acuerdo con datos consultados en el portal educativo Colombia Aprende, adscrito al Ministerio de Educación Nacional, en Colombia había aproximadamente 180 programas virtuales registrados, entre programas técnico-profesionales (48), tecnológicos (54), profesional universitario (37) y posgrados: especializaciones (55) y maestrías (9).

De acuerdo con datos del Ministerio de Educación Nacional, desde 2010 se ha venido registrando una tasa de crecimiento del número de matrículas en educación a distancia y virtual en 
Colombia: 12.000 estudiantes en 2010; 65.000 estudiantes en 2015 y 80.000 estudiantes en el 2017, y de acuerdo con datos del Sistema Nacional de Información de Educación Superior (SNIES), 386 programas de pregrado cuentan con registro calificado en la modalidad a distancia; 422 programas de pregrado tienen registro calificado en la modalidad virtual, y hay 131 programas académicos de posgrado en modalidad a distancia y 281 en modalidad virtual con registro calificado. Con respecto a las licenciaturas en el país, El SNIES registra 64 licenciaturas en Colombia en modalidad a distancia, activas y con registro calificado, de las cuales 11 tienen acreditación de alta calidad, y 17 virtuales, que no cuentan con acreditación de alta calidad [3].

En Colombia, las entidades que se han encargado de orientar políticas y lineamientos para la incorporación de las TIC a los procesos formativos y educativos son el Ministerio de Educación Nacional, el Ministerio de las Tecnologías de la Información y las Comunicaciones, la organización Virtual Educa y la red Renata, además se reconoce la participación de instituciones como la Red de Instituciones Técnicas, Profesionales Tecnológicas y Universitarias Públicas (Redttu), la Asociación Colombiana de Universidades (Ascun), la Asociación Colombiana de Instituciones de Educación Superior con Educación Formación técnica Profesional y/o Tecnológica (Aciet), la Asociación Colombiana de Instituciones de Educación Superior con Programas a Distancia (Acesad) y su versión internacional: La Asociación Iberoamericana de Educación Superior a Distancia (Aiesad), y el conjunto de instituciones de educación superior que se ha vinculado a estas organizaciones o han incursionado en estas modalidades educativas; incluso varios de los documentos que el Ministerio de Educación Nacional ha socializado para orientar los referentes conceptuales de las mismas y establecer lineamientos de calidad desde 2008, provienen del trabajo en estas organizaciones, particularmente de la Acesad, lo que sumando a la Ley 134 de 2009, "por la cual se definen Principios y conceptos sobre la sociedad de la información y la organización de las Tecnologías de la Información y las Comunicaciones -TIC-, se crea la Agencia Nacional del Espectro y se dictan otras disposiciones», más conocida como Ley de TIC, ha empezado a sustentar el marco general de política y regulación de dichas modalidades educativas [11].

\section{DifERENCIAS INTERNA ENTRE LAS MODALIDADES DE EDUCACIÓN A DISTANCIA Y VIRTUAL}

Ahora bien, y ante la necesidad de diferenciar las dos modalidades educativas de la metodología a distancia:

Por su carácter transtemporo-espacial, transfronterizo y global, la modalidad de educación a distancia se define como un conjunto de relaciones pedagógicas entre estudiantes, docentes e institución, basadas o apoyadas en el uso de tecnologías para el desarrollo sistémico de procesos formativos de calidad. Esta modalidad educativa promueve la inclusión y la movilidad social, fundamentada en el aprendizaje autónomo y la autogestión, que utiliza pedagógica y didácticamente diversas metodologías, mediaciones y estrategias, en las que incorpora el uso de medios y tecnologías disponibles y accesibles, para la provisión y certificación del servicio educativo de la formación integral [1].

Su principal característica es la capacidad de romper los límites físicos y temporales propias de la metodología presencial y flexibilizar el acceso a la educación sobre todo para grupos poblacionales tradicionalmente vulnerables a factores de exclusión, por su ubicación espacial o por su vinculación a lógicas de producción y trabajo. En Colombia, por ejemplo, en 2015, el país alcanzó una tasa de cobertura del $49.4 \%$ en el nivel de educación superior; pero, aunque la oferta se presenta, en todos los departamentos, se concentra mayoritariamente en determinadas regiones (Bogotá, Antioquia, Valle del Cauca, Atlántico y Santander), y en las zonas rurales existe una falta de articulación entre la educación secundaria y media, y la educación superior, "en Colombia hay alrededor de 740 mil jóvenes que residen en zonas rurales dispersas, de los cuales 273 mil jóvenes entre los 17 y los 25 años finalizaron bachillerato $\left(\right.$ grado $\left.11^{\circ}\right)$. De estos, cerca de $84 \mathrm{mil}$ (31\%) afirmaron estar asistiendo a una institución de educación superior, mientras que, de los 189 mil restantes, cerca de 147 mil $(78 \%)$ reportaron no haber aprobado ningún año de educación técnica, tecnológica o universitaria" [11].

La diferencia entre las dos modalidades radica fundamentalmente en la importancia de las tecno- 
logías digitales de la comunicación y la educación que en la modalidad virtual es central y en la modalidad a distancia tradicional son un recurso que se suma a los demás medios tecnológicos; por ejemplo, el uso de plataformas educativas como Moodle, es indispensable en la modalidad virtual, pero puede ser subsidiario en la modalidad de distancia tradicional; también se diferencian por los roles de los actores educativos, dado que en la educación a distancia tradicional la relación docente-estudiante es fundamental para la formación integral, por lo que tienen porcentajes más altos de encuentros presenciales, en tanto que en la virtual la relación puede la relación o mediatizarse aún más a través de las plataformas y ambientes virtuales de aprendizaje, a partir de la incorporación del rol de tutor, entendido como persona encargada de hacer seguimiento y monitoreo del desempeño de los estudiantes y la ejecución de las actividades programadas en los entornos virtuales de aprendizaje, producidos por los docentes. En la modalidad a distancia tradicional esta figura no existe, aunque se puedan incorporar recursos de LMS para apoyar los procesos formativos y el seguimiento que hacen los docentes del desempeño de los estudiantes.

El siguiente cuadro resume las principales diferencias entre estas modalidades (ver Tabla I).
La educación virtual se denomina e-learning porque en esta modalidad de enseñanza-aprendizaje el proceso pedagógico se encuentra apoyado en el uso de las TIC, uso vinculado específicamente a la conectividad entre computadores de tal forma que el estudiante puede interactuar con un contenido educativo, el docente interactúa con el alumno, a través de una plataforma electrónica, implementada a través de internet, más específicamente de la web [13].

En término generales las características educativas del e-learning son: i) funciona en la red que requiere para la publicación, actualización, almacenamiento, recuperación y distribución, en tiempo real, de las actividades, los materiales educativos y la información, de un curso o proceso de formación, ii) los elementos del diseño educativo son entregados al estudiante mediante el uso de computadores conectados a la red a través de tecnología estándar de internet, iii) se clasifica en de acuerdo con el tipo de comunicación, en sincrónico y asincrónico [13]. En el primero se emula el proceso educativo que se da al interior de un salón de clase, utilizando las tecnologías del internet; por lo tanto, requiere que todos los participantes estén presentes al mismo tiempo, en este caso, conectados a la red en forma concurrente; el segundo, se basa en acciones de los participantes, diferidas en el tiempo.

Tabla I. Diferencias entre la modalidad virtual y modalidad a distancia tradicional

\begin{tabular}{|c|c|}
\hline Distancia Virtual & Distancia Tradicional \\
\hline $\begin{array}{l}\text { - También conocida como Online } \\
\text { - Desarrolla programas de formación de enseñanza-aprendizaje a } \\
\text { través del Ciberespacio. } \\
\text { - Utiliza una mediación que no requiere que el cuerpo, tiempo y } \\
\text { espacio se conjuguen para lograr establecer un encuentro de } \\
\text { diálogo o ambientes de aprendizaje. } \\
\text { - Acción que busca propiciar espacios de formación apoyándose } \\
\text { en las TIC para instaurar una nueva forma de enseñar y de } \\
\text { aprender. } \\
\text { - Es una modalidad de la educación a distancia; implica una } \\
\text { nueva visión de las exigencias del entorno económico, social y } \\
\text { político, así como de las relaciones pedagógicas y de las TIC. } \\
\text { - El Tutor B interactúa para orientar los procesos de aprendizaje y } \\
\text { resolver, en cualquier momento y de forma más rápida, las } \\
\text { inquietudes de los aprendices. }\end{array}$ & $\begin{array}{l}\text { - Utiliza todos los recursos de la educación } \\
\text { virtual / Online. } \\
\text { - Interactúa alumno-profesor, alumno-alumno, } \\
\text { profesor-alumno, alumno-profesor-alumno, a } \\
\text { través de entornos presenciales y virtuales. } \\
\text { - Utiliza diversos medios educativos para el } \\
\text { proceso de enseñanza-aprendizaje. } \\
\text { - Desarrolla los programas de formación a través } \\
\text { de las TIC y sin ellas. Por ello, combina la } \\
\text { enseñanza cara a cara y virtual. } \\
\text { - Los materiales didácticos son el soporte básico } \\
\text { de transmisión de la información. }\end{array}$ \\
\hline
\end{tabular}

Fuente: MEN, 2016, p. 28 [12] 
De acuerdo con la frecuencia, los recursos y el uso de las TIC, el e-learning se clasifica en las modalidades de totalmente en línea (o completamente e-learning), semipresencial (o blended-learning) que combina la formación online con seciones presenciales; mobil-learning (aprendizaje electrónico móvil) que es la conjunción entre el e-learning y el acceso a la información desde cualquier lugar, por medio de aparatos de tecnología móvil como el celular o las agendas electrónicas; ulearning: es la combinación entre el mobil-learning y el e-learning; we-learning, que consiste en un tipo de educación a distancia que se produce entre iguales, en el que se comparte conocimiento, por medio de nuevas herramientas colaborativas que brinda la web [14]. El b-learning (blendedlearning) ha sido el más acogido por las comunidades educativas que buscan un proceso de aprendizaje mixto, más complejo, colaborativo y de naturaleza semipresencial.

\section{LOS INDICADORES DE EVALUACIÓN DE LA CALIDAD DE LAS MODALIDADES EDUCATIVAS A DISTANCIA Y VIRTUAL}

La propuesta de educación a distancia y virtual en Colombia se ha orientado principalmente desde Ministerio de Educación, que en su calidad de entidad promotora y normativa en cuanto al ámbito educativo se refiere, se ha encargado de generar procesos y estrategias para que este tipo de educación se implemente en el país. Para el Ministerio de Educación este proceso debe comenzar a desarrollarse desde todas y cada una de las instituciones de educación superior, que en su calidad de innovadoras deben fomentar y ampliar el uso de las nuevas tecnologías dentro del aula de clases. Se considera que, implementando la TIC en los procesos formativos, se sientan las bases para aumentar la oferta de educación a distancia y virtual, con lo cual se ampliarán las oportunidades y beneficios a los colombianos que, por diversas razones, no han tenido acceso directo a la educación superior [15].

Desde el Ministerio de Educación Nacional, el Consejo Nacional de Acreditación (CNA), el Sistema de Aseguramiento de la Calidad de la Educación Superior (Saces) y la Comisión Nacional Intersectorial de Aseguramiento de la Calidad de la Educación (Conaces), como entidades encarga- das de evaluar los programas de pregrado y posgrado de las instituciones de educación superior y el cumplimiento de los requisitos para la creación y permanencia de programas de educación superior han venido determinando los lineamientos de calidad de los programas de educación a distancia y virtual en Colombia que se desprenden de la normatividad nacional.

En 2006, el Ministerio de Educación Nacional público el primer documento de Indicadores para la autoevaluación con fines de acreditación de programas de pregrado en las modalidades a distancia y virtual de los primeros lineamientos; en 2010, el documento Lineamientos para la educación virtual en la educación superior y, en 2013, el documento Lineamientos de calidad para la verificación de las condiciones de calidad de los programas virtuales y a distancia, a partir de los cuales se han establecido las condiciones para la aprobación de programas académicos en estas modalidades, para el aseguramiento de la calidad y para la obtención de la acreditación de alta calidad para los mismos. Estos lineamientos orientan la evaluación de factores como la denominación y justificación de estos programas, los aspectos curriculares, la organización de las actividades de formación por créditos académicos, la formación investigativa, la proyección social, la selección y evaluación de estudiantes, el personal académico, los medios educativos, la infraestructura, la estructura académico-administrativa, la autoevaluación, las políticas y estrategias de seguimiento a egresados, el bienestar universitario y los recursos financieros. La propuesta del Decreto 1280 del 25 de julio de 2018, establecía, para el registro calificado de los programas a distancia y virtuales:

Para obtener el registro calificado de los programas a distancia y virtuales, las instituciones, además de mostrar el cumplimiento de las condiciones de calidad establecidas en el presente Capítulo y orientarse por los Lineamientos Específicos para esta modalidad, deben informé la manera en que desarrollarán las actividades de formación académica, la utilización efectiva de mediaciones pedagógicas y didácticas y el uso de formas de interacción apropiadas, que apoyen y fomenten el desarrollo de competencias para el aprendizaje autónomo; el soporte institucional para procesos académicos, administrativos y tecnológicos; y el 
personal docente calificado específicamente para la modalidad y las estrategias de evaluación y seguimiento de resultados de aprendizaje que sean pertinentes. Los programas a distancia (tradicional y virtual) deben tener, al momento de solicitar el registro calificado, el $25 \%$ de los créditos virtuales completamente desarrollados. Estos créditos deben corresponder a los cursos de formación disciplinar. Para la renovación de estos programas, debe presentarse el $100 \%$ de los cursos desarrollados, la evaluación hecha por los estudiantes de cada curso realizado, el análisis de ésta y las estrategias de mejoramiento derivadas de este análisis, si hay lugar a ello. En los programas a distancia (tradicional y virtual) la institución debe indicar cómo llevará a cabo el proceso de diseño, gestión, producción, distribución y uso de materiales y recursos, con observancia de las disposiciones que salvaguardan los derechos de autor (Artículo 2.5.3.2.2.9.3.)

\section{LAS COMPETENCIAS DE TIC}

Uno de los retos más importante para la incorporación de estas modalidades educativas en las instituciones de educación superior ha sido la capacitación de los docentes, quienes no solo se encargan de la implementación de estas modalidades educativas, sino que también, son los encargados de promover, generar y promulgar nuevas estrategias de enseñanza-aprendizaje con ayuda las TIC. Por lo anterior, el Ministerio de Educación Nacional ha establecido una serie de derroteros en relación con las competencias en el uso de las TIC para los docentes, en un documento titulado Competencias TIC para el desarrollo profesional docente. Estas orientaciones buscan fomentar, en los docentes, la innovación educativa y la transformación de las prácticas educativas mediante la integración pertinente de las TIC [16].

Según el MEN, formar a los docentes para la innovación educativa debe tener como finalidad prepararlos para "aportar a la calidad educativa mediante la transformación de las prácticas educativas con el apoyo de las TIC, adoptar estrategias para orientar a los estudiantes hacia el uso de las TIC para generar cambios positivos sobre su entorno, y promover la transformación de las instituciones educativas en organizaciones de aprendizaje" [16].
La innovación educativa con el uso de las TIC parte de considerar que estas pueden ser utilizadas para mejorar los procesos de enseñanza-aprendizaje y sus métodos y, en este sentido se la define como:

Un proceso en el que la práctica educativa, con la mediación de TIC, se reconfigura para dar respuesta a una necesidad, expectativa o problemática, desde lo que es pertinente y particular de un contexto, propiciando la disposición permanente al aprendizaje y la generación de mejores condiciones en las realidades de los actores educativos. La innovación educativa cobra relevancia en su uso, apropiación y difusión en la, y por la comunidad educativa» [16].

Las competencias para el desarrollo de la innovación educativa apoyada por las TIC son: tecnológica, comunicativa, pedagógica, investigativa y de gestión. La competencia tecnológica se entiende como la capacidad para seleccionar y utilizar de forma pertinente, responsable y eficiente una variedad de herramientas tecnológicas entendiendo los principios que las rigen, la forma de combinarlas y las licencias que las amparan; la comunicativa, como la capacidad para expresarse, establecer contacto y relacionarse en espacios virtuales y audiovisuales a través de diversos medios y con el manejo de múltiples lenguajes, de manera sincrónica y asincrónica; la pedagógica, como la capacidad de utilizar las TIC para fortalecer los procesos de enseñanza y aprendizaje, reconociendo alcances y limitaciones de la incorporación de estas tecnologías en la formación integral de los estudiantes y en el propio desarrollo profesional de los docentes; la investigativa, como la capacidad de utilizar las TIC para la transformación del saber y la generación de nuevos conocimientos y la de gestión, como la capacidad para utilizar las TIC en la planeación, organización, administración y evaluación de manera efectiva de los procesos educativos; tanto a nivel de prácticas pedagógicas como de desarrollo institucional [16].

Por otra parte, las competencias se desarrollan y evidencian en diferentes niveles o grados de complejidad y especialización, esto son: exploración, integración e innovación.

El primer nivel o momento de exploración, se caracteriza por permitir el acercamiento a un con- 
junto de conocimientos que se constituyen en la posibilidad para acceder a estados de mayor elaboración conceptual. En el segundo nivel o momento de integración, se plantea el uso de los conocimientos ya apropiados para la resolución de problemas en contextos diversos. Finalmente en el tercer nivel o momento de innovación, se da mayor énfasis a los ejercicios de creación; lo que permite ir más allá del conocimiento aprendido e imaginar nuevas posibilidades de acción o explicación [16].

Estas competencias y sus niveles de especialización se proponen como comunes a la formación de docentes; pero, en las modalidades a distancia y virtual no solo resultan necesarias sino indispensables para los procesos de enseñanza y aprendizaje, el diseño curricular y las implicaciones de la inclusión de las TIC en los procesos educativos para diseñar ambientes de aprendizaje que respondan a las necesidades particulares de los entornos sociales; todo lo cual se constituye en las bases para el logro de las finalidades del uso de las TIC en el desarrollo profesional docente: una uso educativo de las TIC pertinente, que atiende a las necesidades de los actores del proceso de enseñanza-aprendizaje y del contexto cultural en el que se desenvuelven; práctico, mediante la utilización directa del TIC de manera vivencial y en situaciones cotidiana; situado, para reconocer las necesidades de los actores del proceso formativo y transformar las prácticas educativas; colaborativo, facilitando y propiciando el aprendizaje conjunto, la creación colectiva, el intercambio entre pares y la participación en redes y comunidades de práctica, e inspirador, que promueve la imaginación, el pensamiento crítico, la creatividad, el desarrollo de talentos y el deseo de aprender a aprender [16].

En cualquiera de sus modalidades, están competencias determinan condiciones de calidad, no solo del trabajo de los docentes, sino también de los procesos formativos en los que estos participan.

\section{LOS MODELOS DE INCORPORACIÓN DE LAS TIC EN LAS UNIVERSIDADES}

La incorporación de las TIC en los procesos educativos en las universidades ha dependido de factores como el nivel de uso, determinado por la función que estas cumplen que puede ser de inte- gración en los procesos formativos, de complementariedad en las estrategias pedagógicas o de sustitución de la modalidad presencial por modalidades blended y e-learning [17].

Estos modelos se sustentan en las misiones y los proyectos educativos institucionales (PEI), así como en las metodologías que estos proyectos definen en los procesos formativos, y en general puede hablarse de cuatro tipos: modelo de campus virtual, modelo presencial con programas virtuales, modelo de campus presenciales con cursos virtuales y modelo de campus presencial con elementos TIC de apoyo [17].

El primero es el que incorporan las universidades que se han constituido como campus virtuales que ofrecen programas e-learning para una población de docentes y estudiantes distribuidos geográficamente, por ejemplo, la Universidad Nacional Abierta y a Distancia (UNAD); el modelo de campus presencial con programas virtuales, ofrece programas presenciales y programas virtuales blended y e-learning, pero conserva la condición institucional presencial; el modelo de campus presencial con cursos virtuales se apoya en la oferta de programas académicos que combinan asignaturas presenciales con asignaturas virtuales, y el modelo de campus presencial con elementos TIC de apoyo, combina metodologías y didácticas pedagógicas en un mismo ambiente de aprendizaje, basado esencialmente en la presencialidad, integrando sus recursos a los contenidos programáticos y objetivos formativos.

\section{CONCLUSIONES}

Uno de los desafíos más importantes para definir programas en estas modalidades es reconocer sus diferencias para determinar no solo las estrategias de aseguramiento de calidad, sino para identificar los indicadores con los cuales se miden.

Con base en la revisión de sus diferencias en este artículo, podemos afirmar que su principal diferencia radica, fundamentalmente, en la importancia de las tecnologías digitales de la comunicación y la educación, que en la modalidad virtual es central y en la modalidad a distancia tradicional 
son un recurso que se suma a los demás medios tecnológicos.

Así, el uso de plataformas educativas como Moodle, es indispensable en la modalidad virtual, pero puede ser subsidiario en la modalidad de distancia tradicional; también se diferencian por los roles de los actores educativos, dado que en la educación a distancia tradicional la relación docenteestudiante es fundamental para la formación integral, por lo que tienen porcentajes más altos de encuentros presenciales, en tanto que en la virtual la relación puede la relación o mediatizarse aún más a través de las plataformas y ambientes virtuales de aprendizaje, a partir de la incorporación del rol de tutor, entendido como persona encargada de hacer seguimiento y monitoreo del desempeño de los estudiantes y la ejecución de las actividades programadas en los entornos virtuales de aprendizaje, producidos por los docentes. En la modalidad a distancia tradicional esta figura no existe, aunque se puedan incorporar recursos de LMS para apoyar los procesos formativos y el seguimiento que hacen los docentes del desempeño de los estudiantes.

\section{REFERENCIAS}

[1] R. Salazar y A. Melo, lineamientos conceptuales de la modalidad de educación a distancia. En N. Arboleda y C. Rama (eds.). La educación superior a distancia y virtual en Colombia: nuevas realidades (pp. 81-112). Bogotá. 2013.

[2] Colombia, Presidencia de la República. Decreto 1075 de 2015, por el cual se expide el Decreto Único Reglamentario de la Educación Superior del Sector Educación. Recuperado de http://www. suin-juriscol.gov.co/viewDocument.asp?ruta= Decretos/30019930. 2015.

[3] Colombia, Ministerio de Educación Nacional. Propuesta de decreto 1280 de 2018, "por el cual se reglamenta el Sistema de Aseguramiento de la Calidad de la Educación Superior, el registro calificado de que trata la Ley 1188 de 2008 y los artículos 53 y 54 de la Ley 30 de 1992 sobre acreditación, por lo que se subrogan los Capítulos 2 y 7 del Título 3 de la Parte 5 del Libro 2 del Decreto 1075 de 2015 -Único Reglamentario del Sector Educación". Recuperado de http:/ / es.presidencia.gov.co/normativa/normativa/DECRETO $\% 201280 \%$ 20DEL $\% 2025 \% 20$ DE $\%$ 20JULIO\%20DE\% 202018. pdf. 2018.
[4] N. Lobo y V. Fallas. La benemérita Universidad Estatal a Distancia en la sociedad del conocimiento. Costa Rica: UNED. 2018.

[5] Colombia, Ministerio de Educación Nacional. Lineamientos para la educación virtual en la educación superior. Recuperado de http:/ / aprende. colombiaaprende.edu.co/ckfinder/userfiles/ files/Lineamientos_para_la_educacion_Virtual_ dic_29.pdf. 2010.

[6] Organización para la Cooperación y el Desarrollo Económicos. Educación a distancia en la educación superior en América Latina. Recuperado de https://read.oecd-ilibrary.org/education/laeducacion-a-distancia-en-la-educacion-superioren-america-latina_9789264277977-es\#page1. 2017.

[7] L. García-Aretio, Historia de la educación a distancia. Ried, 1, (2), 8-27. 1999.

[8] M. Arboleda, Diagnóstico estadístico y Tendencias de la Educación Superior a Distancia en Colombia. En N. Arboleda y C. Rama (eds.). La educación superior a distancia y virtual en Colombia: nuevas realidades (pp. 31-46). Bogotá. 2013.

[9] A. Facundo, El difícil tránsito a la virtualidad. La educación superior a distancia en Colombia luego de tres décadas de desarrollo. En C. Rama y J. Pardo. La educación superior a distancia: Miradas diversas desde Iberoamérica (pp. 55-77). Recuperado de http://virtualeduca.org/documentos/ observatorio/oevalc_2010_(miradas).pdf. 2010.

[10] B. Giraldo y L. Cuartas, “E-learning: aprendizaje a través de Internet", documento disponible en http: // biblouniandes.blogspot.com/2008/11/ensayoaprendizaje-travs-del-internet.html. 2008.

[11] Colombia, Ministerio de Educación Nacional. Plan especial para la educación rural: hacia el desarrollo rural y la construcción de paz. Recuperado de file:/ / C:/Users/segon/Desktop/CC\%20 Concepto/Men\%20peer.pdf. 2017.

[12] Colombia, Ministerio de Educación Nacional. Lineamientos con condiciones específicas de calidad para los programas ofrecidos en las modalidades virtual y combinada (documento de trabajo). Recuperado de www.redttu.edu.co/.../2016/.../MEN-Linea mientos_Educacion_Distancia_Version1_M. 2016.

[13] F. González, "Tendencias en e-learning: modalidades, barreras y futuro del e-learning". Disponible en www.articulo.org/articulo/608/tenden cias_en_elearning_modalidades_barreras_y_ futuro_del_elearning.html. 2007.

Rev. Ingeniería, Matemáticas y Ciencias de la Información Vol. 8 / Núm. 16 / julio - diciembre de 2021; pág. 97-107 
[14] M. Fernández, M. Hacia un elearning 2.0+. Disponible en: es.slideshare.net/montsefc/hacia-unelearning-20. 2008.

[15] A. Alvarado, lineamientos conceptuales de la modalidad de educación a distancia. En N. Arboleda y C. Rama (eds.). La educación superior a distancia y virtual en Colombia: nuevas realidades (pp. 81-112). Bogotá. 2013.
[16] Colombia, Ministerio de Educación Nacional. Competencias TIC para el desarrollo profesional docente. Recuperado de https://www.mine ducacion.gov.co/1621/articles-339097_archivo_ pdf_competencias_tic.pdf. 2013.

[17] L. Osorio, M-F. Aldana y D. Leal, Incorporación de TIC en ambientes de aprendizaje en educación superior. Disponible en http:/ / www.diegoleal.org/ docs / 2006/ Articles/Teledu2006-Experiencia AVA.pdf. 2006. 
